\title{
INIBIÇÃO DO DESENVOLVIMENTO FÚNGICO ATRAVÉS DA UTIĻIZAÇÃO DE ÓLEOS ESSENCIAIS DE CONDIMENTOS
}

\author{
Inhibition fungi growth through of utilization essential oils of spice
}

\author{
Marcelo Cláudio Pereira ${ }^{1}$, Georgia Rocha Vilela ${ }^{2}$, Lívia Martinez Abreu Soares Costa ${ }^{3}$, \\ Reginaldo Ferreira da Silva ${ }^{4}$, Anderson Felicori Fernandes ${ }^{5}$, \\ Ellen Waleska Nascimento da Fonseca ${ }^{6}$, Roberta Hilsdorf Piccoli ${ }^{7}$
}

\begin{abstract}
RESUMO
Objetivou-se com este trabalho avaliar os efeitos inibitórios, "in vitro", de óleos essenciais dos condimentos, alecrim (Rosmarinus officinalis L.), cebola (Allium cepa L.), manjericão (Ocimum basilicum L.), menta (Mentha piperita L.) e orégano (Origanum vulgare L.), sobre o desenvolvimento de fungos. Os óleos foram extraídos pela técnica de arraste a vapor e testados nas concentrações de 500; 1000; 1500 e $2000 \mathrm{mg} / \mathrm{mL}^{-1}$. Como culturas de teste foram utilizados os fungos Fusarium sp.; Aspergillus ochraceus Wilhelm.; Aspergillus flavus Link e Aspergillus niger van Tieghem obtidos da micoteca do EcoCentro/EPAMIG em Lavras, MG. O óleo essencial do orégano inibiu o desenvolvimento dos fungos testados em todas as concentrações exceto o fungo $A$. niger que teve o seu desenvolvimento micelial inibido a partir da concentração de $1000 \mathrm{mg} / \mathrm{mL}^{-1}$,. Os óleos de alecrim, menta, cebola e manjericão tiveram um efeito pronunciado a partir da concentração de $1500 \mathrm{mg} / \mathrm{mL}^{-1}$.
\end{abstract}

Termos para indexação: Condimentos, óleos essenciais, fungos, inibição.

\begin{abstract}
The objective of this research was to evaluate "in vitro" effect of essential oils of the condiments, rosemary (Rosmarinus officinalis L. ), onion (Allium cepa L.), basil (Ocimum basilicum L.), mint (Mentha piperita L.) and oregano (Origanum vulgare L.) about fungi development. The essential oils were extracted by utilizing the vapor "dragging" technique and tested in the concentrations of 500; 1000; 1500 and $2000 \mathrm{mg} / \mathrm{mL}^{-1}$. The fungi Fusarium sp; Aspergillus ochraceus Wilhelm; Aspergillus flavus Link and Aspergillus niger van Tieghem were obtained from the Fungal Culture Collection, EcoCentro/EPAMIG in Lavras, MG. The essential oil oregano inhibited completely the tested fungi development except the fungus A. niger that had its development inhibited starting from the concentrations of $1000 \mathrm{mg} / \mathrm{mL}^{-1}$. The rosemary, mint, onion and basil oils presented a pronounced effect starting from the concentration of $1500 \mathrm{mg} / \mathrm{mL}^{-1}$.
\end{abstract}

Index terms: Condiments, essential oils, fungi, inhibition.

(Recebido para publicação em 2 de março de 2005 e aprovado em 28 de julho de 2005)

\section{INTRODUÇÃO}

Por definição, condimentos e especiarias são produtos aromáticos de origem vegetal empregados principalmente para conferir sabor aos alimentos. Além desta utilidade possuem também propriedades antimicrobianas, antioxidantes e medicinais e existem aproximadamente 70 condimentos diferentes, cultivados e utilizados em todo mundo (SHELEF, 1983).

Segundo o mesmo autor, os condimentos e as especiarias foram amplamente utilizados por civilizações antigas para melhorar a palatabilidade de alimentos e bebidas. Os egípicios aproveitavam também as características preservativas destes temperos; as propriedades dos óleos essenciais de cravo, canela e cássia foram empregadas no processo de mumificação de seus mortos. Atualmente, vários óleos e extratos com alta atividade bactericida e fungicida, extraídos de ervas e outras plantas têm sido utilizados em hospitais e, particularmente, em salas de cirurgia no processo de desinfecção.

A utilização de substâncias naturais, de origem vegetal, torna o alimento mais atrativo ao consumidor por não apresentarem efeito tóxico, mesmo quando empregadas em concentrações relativamente elevadas. Além dos benefícios proporcionados à saúde, diversos estudos têm demonstrado o efeito inibidor de condimentos no desenvolvimento de microrganismos deterioradores e

\footnotetext{
${ }^{1}$ Biólogo Doutorando em Ciência dos Alimentos área de atuação Microbiologia de Alimentos/DCA, Universidade Federal de Lavras/UFLA - Cx. P. 3037 37200-000 - Lavras/MG - marcelo.claudio@posgrad.ufla.br

${ }^{2}$ Engenheira Agrônoma, Mestranda em Tecnologia de Alimentos/ESALQ.

${ }^{3}$ Engenheira Agrônoma, Mestranda em Ciência dos Alimentos/UFLA - Cx. P. 3037 - 37200-000 - Lavras/MG.

${ }^{4}$ Doutorando em Ciência dos Alimentos/DCA - UFLA - Cx. P. 3037 - 37200-000 - Lavras/MG.

5 Mestrando em Ciência dos Alimentos/DCA - UFLA - Cx. P. 3037 - 37200-000 - Lavras/MG.

${ }^{6}$ Mestranda em Ciência dos Alimentos/DCA - UFLA - Cx. P. 3037 - 37200-000 - Lavras/MG.

${ }^{7}$ Professora Adjunto, Departamento de Ciência dos Alimentos da Universidade Federal de Lavras/UFLA - Cx. P. 3037 - $37200-000$ - Lavras/MG.
} 
patogênicos veiculados por alimentos. Existe também a perspectiva de substituir os aditivos sintéticos por conservantes naturais presentes nos condimentos (BARA, 1992). Deans \& Ritchie (1987) afirmam que a substituição de aditivos sintéticos por naturais, dependerá fundamentalmente da determinação de uma concentração ideal e, segundo Shelef (1983), as concentrações normalmente empregadas para realçar o aroma e sabor que variam de 0,5 a $1 \%$ não inibem o desenvolvimento microbiano que depende de concentrações superiores a $1 \%$.

Bullerman (1974) analisando o desenvolvimento de fungos em pães brancos e adicionados de uva-passa e canela, verificou neste último uma inibição acentuada de micotoxinas e do desenvolvimento micelial de Aspergillus parasiticus Speare, chegando a $100 \%$ quando foi utilizado extrato alcóolico de canela a $20 \%$, já em concentrações menores o extrato de canela foi mais eficiente na inibição de micotoxinas do que no desenvolvimento micelial do fungo. Bullerman el al. (1977) observaram que os óleos essenciais de canela a $200 \mathrm{mg} / \mathrm{mL}$ e de cravo a $250 \mathrm{mg} / \mathrm{mL}$ foram inibidores do desenvolvimento e da produção de toxina de A. parasiticus, enquanto que o aldeído cinâmico e o eugenol, principais constituintes dos seus óleos essenciais de canela e cravo, respectivamente, apresentaram efeito inibidor a 150 e $125 \mathrm{mg} / \mathrm{mL}$, respectivamente e concluíram que estes são os compostos de maior atividade antifúngica.

Vários estudos têm comprovado o efeito de compostos isolados extraídos de óleos essenciais de plantas que atuam como fungicidas naturais inibindo a atividade fúngica e, um número significativo destes constituintes tem se mostrado eficaz (CHAO \& YOUNG, 2000).

Hitokoto et al. (1980) testaram 29 condimentos e observaram uma inibição completa de três espécies toxigênicas de Aspergillus por extratos de cravo, semente de anis e pimenta, enquanto que os outros condimentos foram eficientes somente na inibição da aflatoxina. $\mathrm{O}$ eugenol e o timol extraídos respectivamente do cravo e do tomilho causaram inibição completa no desenvolvimento de Aspergillus flavus e A. versicolor (Vuill) Tirab a $0,4 \mathrm{mg}$ / $\mathrm{mL}$ ou menos; na concentração de $2 \mathrm{mg} / \mathrm{mL}$ o anetol extraído das sementes de anis inibiu o desenvolvimento de todas as estirpes testadas. Benjilali et al. (1984) testaram o efeito de 6 óleos essenciais em 39 espécies de fungos do gênero Penicillium, Aspergillus e outros, o óleo de tomilho foi o mais eficiente, seguido de 3 tipos de estragão, alecrim e eucalipto. Farag et al. (1989) obtiveram a seqüência decrescente de maior atividade fungicida de óleos essenciais para A. parasiticus: tomilho, cominho, cravo, alcaravia, alecrim e sálvia.
O óleo essencial de menta e o mentol podem ser usados ainda numa infinidade de outros produtos, pois seu efeito sobre os microrganismos pode ser aproveitado de várias maneiras (MAIA, 1994). Singh et al. (1993) demonstraram o efeito fungicida e fungistático desse óleo sobre 23 espécies, entre elas Alternaria sp, Curvalaria lunata (Wakker) Boedijn, Fusarium moliniforme Sheld. e F. solani Mart. (Sacc), os autores usaram concentrações variando de 500 a $10.000 \mathrm{mg} / \mathrm{mL}$ de óleo de menta nos respectivos meios de cultura, e observaram inibição de $100 \%$ dos micélios, a partir de $2.000 \mathrm{mg} / \mathrm{mL}$, o que levou a afirmar que o óleo de menta (Menta arvensis), devido à sua forte atividade fungicida e largo espectro de atividade, superior a alguns fungicidas comerciais, pode ser usado como um forte produto no controle de doenças de plantas e animais. Singh et al. (1992) concluíram em seus estudos que o óleo de menta, além de antifúngico, desempenha um papel antibacteriano, controlando o desenvolvimento de Salmonella sp e Staphylococcus sp; entre os fungos controlou-se Alternaria sp, Fusarium sp, Sclerotium rolfsii Sacc. e Aspergillus parasiticus. Os autores sugerem o uso direto sobre grãos e alimentos armazenados, visando o controle de microrganismos e insetos.

Chalfoun et al. (2004) avaliaram o efeito "in vitro" de óleos essenciais dos condimentos alho, canela, cravo e tomilho testados em concentrações de 500, 1000, 1500 e $2000 \mathrm{mg} / \mathrm{ml}$, e do óleo de cravo nas concentrações de 200, 400, 600 e $800 \mathrm{mg} / \mathrm{ml}$, sobre o desenvolvimento micelial dos fungos Rhizopus sp., Penicillium spp., Eurotium repens De Bary e Aspergillus niger e constataram uma inibição total do óleo de canela sobre fungos testados, os óleos de tomilho e alho tiveram o mesmo efeito nas concentrações mais altas, o cravo inibiu o desenvolvimento dos fungos a partir da concentração de $600 \mathrm{mg} / \mathrm{ml}$, exceto o fungo Penicillium spp. que foi verificado na concentração de $800 \mathrm{mg} / \mathrm{ml}$.

Com o presente trabalho, objetivou-se determinar o efeito da adição de óleos essenciais em meio sintético sobre o desenvolvimento micelial dos fungos Aspergillus niger, Aspergillus ochraceus, Aspergillus flavus e Fusarium sp.

\section{MATERIAL E MÉTODOS}

\section{Efeito "in vitro" de óleos essenciais sobre o desenvolvimento fúngico.}

O presente trabalho foi desenvolvido no Laboratório do EcoCentro da EPAMIG, localizado no município de Lavras, sul de Minas Gerais.

Ciênc. agrotec., Lavras, v. 30, n. 4, p. 731-738, jul./ago., 2006 
Como culturas de teste foram utilizados gêneros e espécies de fungos, Fusarium sp., Aspergillus ochraceus, A. niger e A. flavus obtidos da micoteca do EcoCentro. O método utilizado foi o bioanalítico "in vitro" observando o desenvolvimento ou inibição dos microrganismos em diferentes concentrações dos óleos usando o meio de cultura BDA (Batata-Dextrose e Agar) apropriado para o desenvolvimento desses fungos. Ao meio de cultura autoclavado por 20 minutos a $120^{\circ} \mathrm{C}$, após o resfriamento, foram adicionados os óleos essenciais que foram diluídos diretamente no meio de cultura nas concentrações de 500,1000, 1500 e 2000 mg/ $\mathrm{mL}$ de meio, preparando-se para cada concentração 200 $\mathrm{mL}$ de meio mais óleo essencial, operações essas realizadas sob condições assépticas (capela de Fluxo Laminar mod. FL7752).

Posteriormente, o meio contendo as diferentes concentrações dos óleos essenciais foi vertido em placas de Petri com $9 \mathrm{~cm}$ de diâmetro e o inóculo, constituído por um disco de micélio de $8 \mathrm{~mm}$ de diâmetro, contendo os fungos em estudo, foram transferidos para o centro das placas. A incubação ocorreu em BOD (mod. 347CDG) com temperatura de $25^{\circ} \mathrm{C}$, e fotoperíodo de 12 horas, durante 7 dias.

Após este período de desenvolvimento foram efetuadas medições ortogonais do diâmetro das colônias tendo como referência o desenvolvimento da placa controle contendo apenas os fungos desenvolvendo sob o meio de cultura sem adição dos óleos essenciais. O esquema fatorial adotado foi com três repetições, em delineamento inteiramente casualizado, obtendo assim, a taxa de desenvolvimento micelial.

Os óleos essenciais que foram testados são os seguintes: alecrim (Rosmarinus officinalis), cebola (Allium cepa $\mathrm{L}$.), manjericão (Ocimum basilicun L.), menta (Mentha piperita L.) e orégano (Origanum vulgari L.).

\section{Obtenção dos óleos essenciais}

Para obtenção dos óleos essenciais das plantas, foi utilizado a metodologia de arraste a vapor. Foram pesados $100 \mathrm{~g}$ dos condimentos, folhas secas (orégano, manjericão, menta e alecrim) e bulbos (cebola) ficando estes em contato direto com 1 litro de água em ebulição, flutuando ou imerso no líquido, dependendo da densidade do material colocado no recipiente de destilação. A água foi posta em ebulição por aquecimento direto conduzindo o vapor d'água, descrita por Koketsu \& Gonçalves (1991).

\section{RESULTADOS E DISCUSSÃO}

De acordo com os resultados apresentados na Tabela 1 e Figuras 1,2,3,4 e 5 observa-se que alguns óleos essenciais obtidos dos condimentos testados apresentaram índices variáveis de inibição do desenvolvimento micelial dos fungos estudados quando comparados com a testemunha não tratada.

Com relação ao orégano, conforme apresentado na Tabela 1 e Figura 2, observa-se que a partir de 500 $\mathrm{mg} / \mathrm{ml}$ todos os fungos estudados tiveram o desenvolvimento significativamente inibido, com exceção do fungo Aspergillus niger, resultado semelhante ao observado por (AKGUL \& KIVANÇ, 1988; VELLUTI et al., 2003, 2004). A redução do desenvolvimento micelial do fungo Aspegillus niger foi obtida a partir da concentração de $1000 \mathrm{mg} / \mathrm{ml}$, resultado este que concorda com aqueles relatados por AZZOUS \& BULLERMAN, 1982; BEUCHAT, 1976; CONNER \& BEUCHAT, 1984, evidenciando o poder antimicrobiano do orégano.

De acordo com a Figura 2, pode-se observar que o óleo essencial de manjericão apresentou um controle do fungo Aspergillus ochraceus a partir da concentração 1000 $\mathrm{mg} / \mathrm{ml}$, em relação aos fungos $A$. niger e $A$. flavus a redução do desenvolvimento micelial foi obtida apenas na concentração de $1000 \mathrm{mg} / \mathrm{ml}$ e o desenvolvimento desses fungos foi estimulado com o aumento das concentrações, resultados semelhantes foram observados por Chalfoun et al. (2004) e Pereira (2001), que relatam que esse tipo de comportamento requer um estudo específico por se tratar de vários fatores tais como: tipo de substrato, reação do fungo ao substrato e diferentes tipos de compostos que podem interferir sobre o comportamento do fungo. $\mathrm{O}$ fungo Fusarium sp. não foi inibido em nenhuma das concentrações testadas.

Os resultados referentes à adição do óleo essencial de alecrim, conforme apresentado na Figura 3, demonstram que em relação ao fungo A. ochraceus, observou-se um tendência de aumento nos índices de inibição do desenvolvimento micelial proporcional ao aumento das concentrações testadas. Os fungos Fusarium sp. e A. flavus tiveram o seu desenvolvimento micelial afetado a partir das concentrações de $1500 \mathrm{e}$ $2000 \mathrm{mg} / \mathrm{ml}$, respectivamente. O fungo $A$. niger não teve o seu desenvolvimento micelial afetado nas concentrações testadas. Vários estudos comprovam as propriedades antimicrobianas deste condimento (BENJILALI et al., 1984; FARAG et al., 1989; SHELEF, 1983). 
TABELA 1 - Eficiência de várias concentrações de óleos essenciais sobre o desenvolvimento micelial de fungos.

\begin{tabular}{|c|c|c|c|c|}
\hline \multirow{2}{*}{ Tratamentos } & \multicolumn{4}{|c|}{ Fungos* } \\
\hline & A. niger & A. flavus & A. ochraceus & Fusarium sp. \\
\hline Orégano $500 \mu \mathrm{g} / \mathrm{ml}$ & $9,0 \mathrm{f}$ & $6,50 \mathrm{~d}$ & $4,53 \mathrm{f}$ & $4,86 \mathrm{~d}$ \\
\hline Orégano $1000 \mu \mathrm{g} / \mathrm{ml}$ & $2,33 \mathrm{c}$ & $3,76 \mathrm{~b}$ & $3,53 \mathrm{c}$ & $2,33 \mathrm{~b}$ \\
\hline Orégano $1500 \mu \mathrm{g} / \mathrm{ml}$ & $0,90 \mathrm{~b}$ & $1,50 \mathrm{a}$ & $2,13 \mathrm{~b}$ & $0,00 \mathrm{a}$ \\
\hline Orégano $2000 \mu \mathrm{g} / \mathrm{ml}$ & $0,00 \mathrm{a}$ & 0,93 a & $0,00 \mathrm{a}$ & $0,00 \mathrm{a}$ \\
\hline Manjericão $500 \mu \mathrm{g} / \mathrm{ml}$ & $9,0 \mathrm{f}$ & $8,23 \mathrm{e}$ & $4,76 \mathrm{~g}$ & $9,0 \mathrm{~g}$ \\
\hline Manjericão $1000 \mu \mathrm{g} / \mathrm{ml}$ & $7,0 \mathrm{e}$ & $7,16 \mathrm{~d}$ & $4,46 \mathrm{f}$ & $9,0 \mathrm{~g}$ \\
\hline Manjericão $1500 \mu \mathrm{g} / \mathrm{ml}$ & $9,0 \mathrm{f}$ & $8,26 \mathrm{e}$ & $4,46 \mathrm{f}$ & $9,0 \mathrm{~g}$ \\
\hline Manjericão $2000 \mu \mathrm{g} / \mathrm{ml}$ & $9,0 \mathrm{f}$ & $7,93 \mathrm{e}$ & $4,46 \mathrm{f}$ & $9,0 \mathrm{~g}$ \\
\hline Alecrim $500 \mu \mathrm{g} / \mathrm{ml}$ & $9,0 \mathrm{f}$ & 8,0 e & $4,60 \mathrm{f}$ & $9,0 \mathrm{~g}$ \\
\hline Alecrim $1000 \mu \mathrm{g} / \mathrm{ml}$ & $9,0 \mathrm{f}$ & $7,70 \mathrm{e}$ & $4,20 \mathrm{e}$ & $8,66 \mathrm{~g}$ \\
\hline Alecrim $1500 \mu \mathrm{g} / \mathrm{ml}$ & $9,0 \mathrm{f}$ & $7,66 \mathrm{e}$ & $4,00 \mathrm{~d}$ & $7,40 \mathrm{f}$ \\
\hline Alecrim $2000 \mu \mathrm{g} / \mathrm{ml}$ & $9,0 \mathrm{f}$ & $7,00 \mathrm{~d}$ & $3,53 \mathrm{c}$ & $6,13 \mathrm{e}$ \\
\hline Menta $500 \mu \mathrm{g} / \mathrm{ml}$ & $9,0 \mathrm{f}$ & $8,20 \mathrm{e}$ & $4,76 \mathrm{~g}$ & $6,93 \mathrm{f}$ \\
\hline Menta $1000 \mu \mathrm{g} / \mathrm{ml}$ & $9,0 \mathrm{f}$ & 8,16 e & $4,80 \mathrm{~g}$ & $4,20 \mathrm{c}$ \\
\hline Menta $1500 \mu \mathrm{g} / \mathrm{ml}$ & $7,0 \mathrm{~d}$ & $8,20 \mathrm{e}$ & $4,80 \mathrm{~g}$ & $9,0 \mathrm{~g}$ \\
\hline Menta $2000 \mu \mathrm{g} / \mathrm{ml}$ & $2,16 \mathrm{c}$ & $5,33 \mathrm{c}$ & $4,73 \mathrm{~g}$ & $9,0 \mathrm{~g}$ \\
\hline Cebola $500 \mu \mathrm{g} / \mathrm{ml}$ & $9,0 \mathrm{f}$ & $8,20 \mathrm{e}$ & $4,70 \mathrm{~g}$ & $9,0 \mathrm{~g}$ \\
\hline Cebola $1000 \mu \mathrm{g} / \mathrm{ml}$ & $9,0 \mathrm{f}$ & $8,16 \mathrm{e}$ & $4,66 \mathrm{~g}$ & $9,0 \mathrm{~g}$ \\
\hline Cebola $1500 \mu \mathrm{g} / \mathrm{ml}$ & $9,0 \mathrm{f}$ & $8,16 \mathrm{e}$ & $4,60 \mathrm{f}$ & $9,0 \mathrm{~g}$ \\
\hline Cebola $2000 \mu \mathrm{g} / \mathrm{ml}$ & $1,90 \mathrm{c}$ & $8,00 \mathrm{e}$ & $4,53 \mathrm{f}$ & $9,0 \mathrm{~g}$ \\
\hline Testemunha & $9,0 \mathrm{f}$ & $8,30 \mathrm{e}$ & $4,90 \mathrm{~g}$ & $9,0 \mathrm{~g}$ \\
\hline $\mathrm{CV}(\%)$ & 6,26 & 6,86 & 1,98 & 4,58 \\
\hline
\end{tabular}

* Médias seguidas pela mesma letra não diferem estatisticamente pelo Teste de Scott \& Knott ao nível de 5\% de probabilidade.

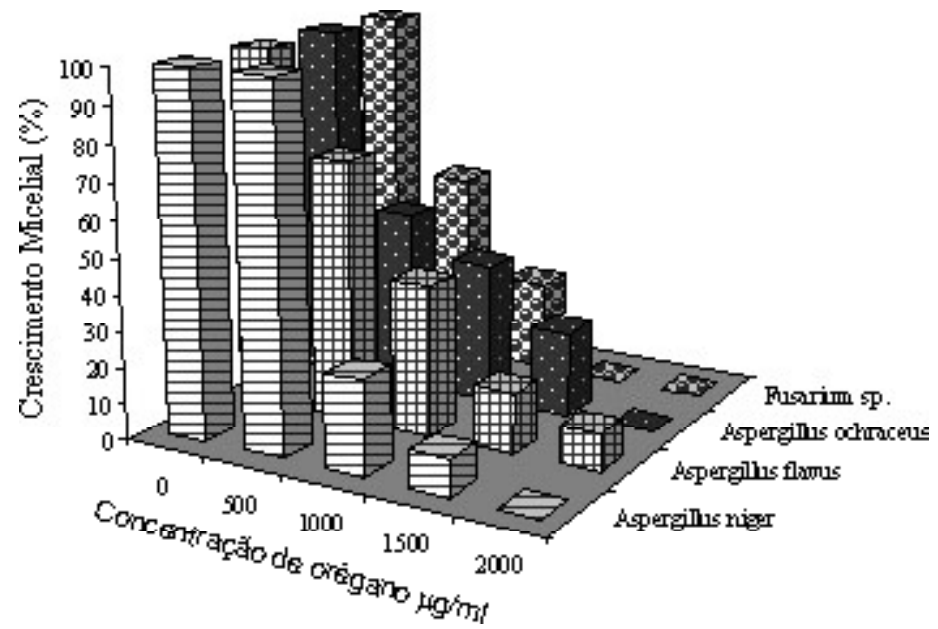

FIGURA 1 -Efeito do óleo essencial de orégano sobre o desenvolvimento micelial dos fungos Fusarium sp., Aspergillus ocrhaceus, A. flavus e A. niger.

Ciênc. agrotec., Lavras, v. 30, n. 4, p. 731-738, jul./ago., 2006 


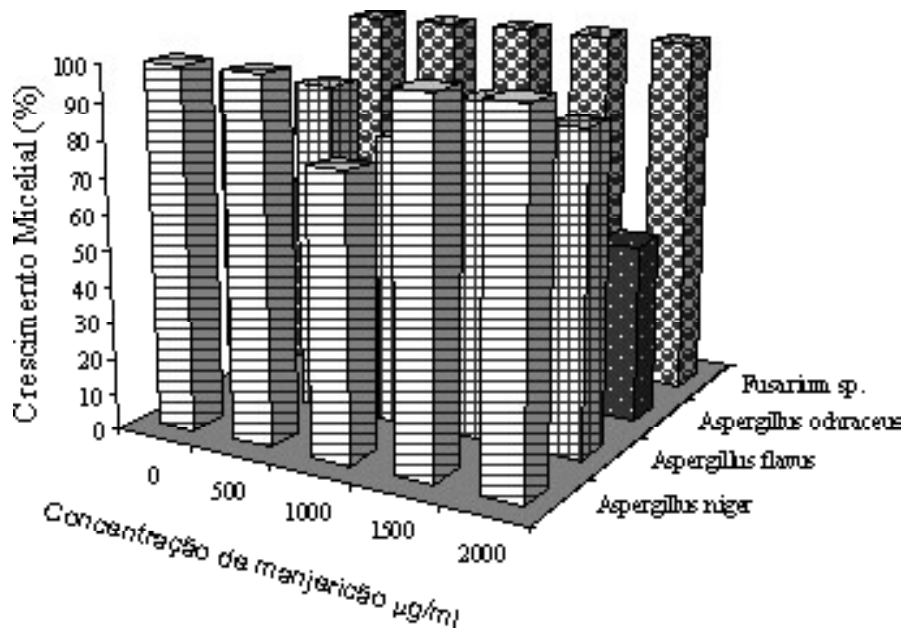

FIGURA 2 - Efeito do óleo essencial de manjericão sobre o desenvolvimento micelial dos fungos Fusarium sp., Aspergillus ocrhaceus, A. flavus e A. niger.

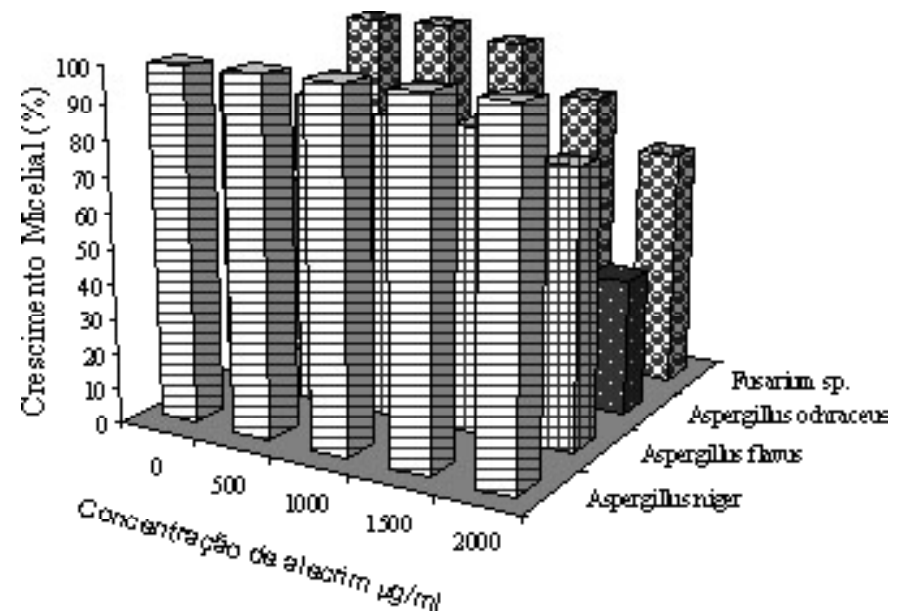

FIGURA 3 - Efeito do óleo essencial de alecrim sobre o desenvolvimento micelial dos fungos Fusarium sp., Aspergillus ocrhaceus, A. flavus e A. niger.

Com relação à atuação do óleo essencial de menta (Figura 4), observou-se uma inibição do desenvolvimento micelial dos fungos A. niger e A. flavus nas concentrações 1500 e $2000 \mathrm{mg} / \mathrm{ml}$, respectivamente. O fungo Fusarium sp. teve o seu desenvolvimento micelial afetado nas concentrações de 500 e $1000 \mathrm{mg} / \mathrm{ml}$, sendo que nas concentrações de 1500 e $2000 \mathrm{mg} / \mathrm{ml}$ o desenvolvimento micelial aumentou acentuadamente, conforme relatado anteriormente este comportamento requer um estudo específico. Em relação ao fungo A. ochraceus não houve diferença estatística em relação à testemunha.

Com relação ao efeito da adição do óleo essecial de cebola (Figura 5), observa-se que não houve inibição do desenvolvimento micelial dos fungos A. flavus e Fusarium sp. em nenhuma das concentrações testadas. Em relação aos fungos A. ochraceus e A. niger estes tiveram o seu desenvolvimento micelial afetado a partir das concentrações de 1500 e $2000 \mathrm{mg} / \mathrm{ml}$, respectivamente, resultados semelhantes foram obtidos por CONNER \& BEUCHAT 1984; DEWIT et al., 1979. 


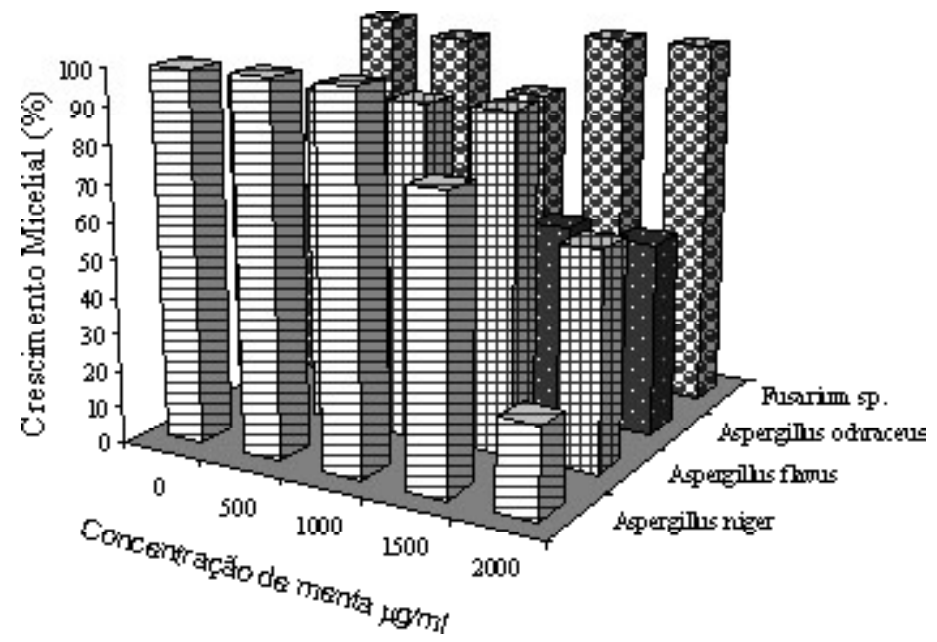

FIGURA 4 -Efeito do óleo essencial de menta sobre o desenvolvimento micelial dos fungos Fusarium sp., Aspergillus ocrhaceus, A. flavus e A. niger.

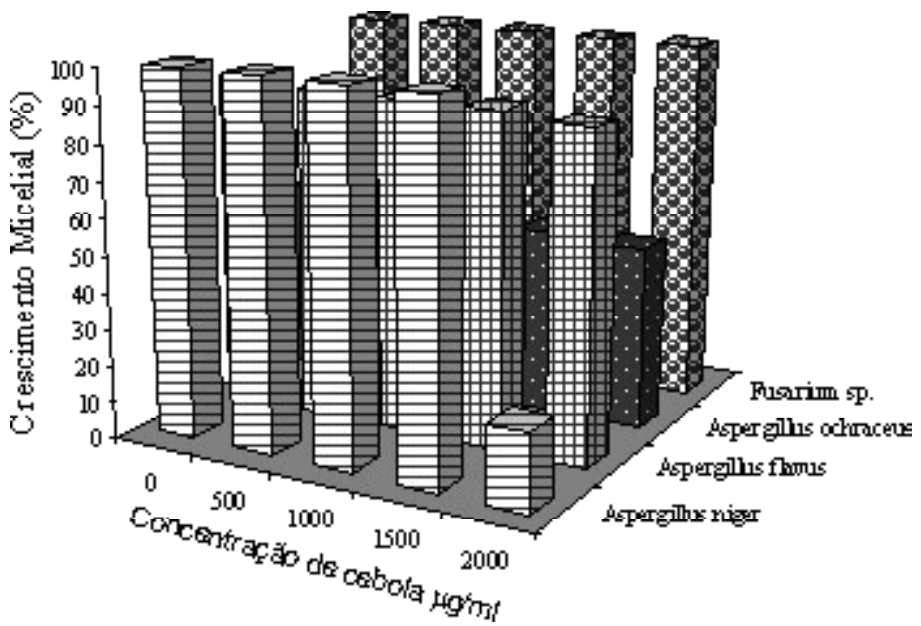

FIGURA 5 - Efeito do óleo essencial de cebola sobre o desenvolvimento micelial dos fungos Fusarium sp., Aspergillus ocrhaceus, A. flavus e A. niger.

\section{CONCLUSÕES}

O ótimo desempenho do óleo essencial de orégano sobre os fungos testados confirma a sua eficiência fungicida e/ou fungistática.

O resultado inferior dos óleos essenciais de cebola, menta, manjericão e alecrim, sobre alguns dos fungos testados pode ser atribuída a uma possível inadequação das doses empregadas, sugerindo que em trabalhos futuros sejam utilizadas doses mais elevadas.

Os óleos essenciais por se tratarem de misturas complexas de diferentes compostos devem ser identificados e testados separadamente com o intuito de elucidar a ação destes compostos sobre o comportamento dos fungos.

\section{REFERÊNCIA BIBLIOGRAFICA}

AKGUL, A.; KIVANÇ, M. Inhibitory effect of selected Turkish spices and oregano components on some common foodborne fungi. International Journal Food Microbiology, Amsterdam, v. 6, p. 263-268, 1988. 
AZZOUS, M. A.; BULlERMAN, L. R. Comparative antimycotic effects of selected herbs, spices, plant components and commercial anti-fungal agents. Journal of Food Protection, Des Moines, v. 45, p. 12981301, 1982.

BARA, M. T. F. Avaliação do efeito inibidor de condimentos no desenvolvimento de Yersinia enterocolitica. 1992. 73 f. Dissertação (Mestrado em Microbiologia Agrícola) - Universidade Federal de Viçosa, Viçosa, 1992.

BENJILALI, B.; TANTAQUI-ELARAKI, A.; AYADI, A.; IHLAL, M. Method to study antimicrobial e effects of essential oils: application to the antifungal activity of six moroccan essences. Journal of Food Protection, Des Moines, v. 47, n. 10, p. 748-752, Oct. 1984.

BEUCHAT, L. R. Sensitivity of Vibrio parahemolyticus to spices and organic acids. Journal Food Science, Chicago, v. 41, p. 899-902, 1976.

BULLERMAN, L. B. Inhibition of aflatoxin production by cinamon. Journal of Food Science, Chicago, v. 39, p. 11631165, 1974.

BULLERMAN, L. B.; LIEW, F. Y.; SEIER, S. A. Inhibition of growth and aflatoxin production by cinamon and clove oils, cinnamic aldehyde and eugenol. Journal of Food Science, Chicago, n. 42, p. 1107-1109, 1977.

CHALFOUN, S. M.; PEREIRA, M. C.; RESENDE, M. L. V.; ANGÉLICO, C. L.; SILVA, R. A. Effect of powdered spice treatments growth, sporulation and production of aflatoxin by toxigenic fungi. Ciência e Agrotecnologia, Lavras, v. 28, n. 4, p. 856-862, jul./ago. 2004.

CHAO, S. C.; YOUNG, D. G. Screening for inhibitory activity of essential oils ou selected bacteria, fungi and viruses. Journal Essentials Oil Research, [S.1.], v. 12, p. 630-649, 2000.

CONNER, D. E.; BEUCHAT, L. R. Effects of essential oils from plants on growth of food spoilage yeasts. Journal Food Science, Chicago, v. 49, p. 429-434, 1984.

DEANS, S. G.; RITCHIE, G. Antibacterial properties of plant essential oils. International Journal of Food Microbiology, Amsterdam, n. 5, p. 165-180, 1987.
DEWIT, J. C.; NOTERMANS, S.; GORIN, N.; KAMPELMACHER, E. H. Effects of garlic and onion oil on toxin production by $\mathrm{C}$. botulinum in meat slurry. Journal Food Protection, Des Moines, v. 42, p. 222-224, 1979.

FARAG, R. S.; DAW, Z. Y.; ABO-RAYA, S. H. Influence of some spice essencial oils on Aspergillus parasiticus growth and production of aflatoxinas in a synthetic medium. Journal of Food Science, Chicago, v. 54, n. 1, p. 54-74, 1989.

HITOKOTO, H.; MOROZUMI, S.; WAUKE, T.; SAKAI, S.; KURATA, H. Inhibitory effects of spices on growth and toxin production of toxigenic fungi. Applied and Evironmental Microbiology, Washington, n. 39, p. 818-822, 1980.

KOKETSU, M.; GONÇALVES, S. L. Óleos essenciais e sua extração por arraste a vapor. Rio de Janeiro: EMBRAPA-CTAA, 1991. 24 p. (Documentos, 8).

MAIA, N. B. Nutrição mineral, desenvolvimento e qualidade do óleo essencial da mentha (Mentha arvensis L.) cultivada em solução nutritiva. 1994. 69 f. Dissertação (Mestrado) - Escola Superior de Agricultura de Luiz de Queiroz, Piracicaba, 1994.

PEREIRA, M. C. Efeito da adição de condimentos no controle de microrganismos, na conservação de produtos de panificação e na inibição de metabólitos produzidos por fungos associados ao café. 2001. 104 p. Dissertação (Mestrado em Ciência dos Alimentos) - Universidade Federal de Lavras, Lavras, 2001.

SHELEF, L. A. Antimicrobial effects os spices. Journal of Food Safety, Westport, n. 6, p. 29-44, 1983.

SINGH, H. N. P.; PRASAD, M. M.; SINHA, K. K. Efficacy of leaf extracts of some medicinal plantas against disease deve lop ment in banana. Letters in Applied Microbiology, Oxford, n. 17, p. 269-271, 1993.

SINGH, S. P.; CHAND, L.; NEGRI, S.; SINGH, A. K. Antibacterial and antifungal activities of Mentha arvensis essential oil. Fitoterapia, [S.1.], v. 63, n. 1, p. 76-78, 1992.

VELLUTI, A.; MARIN, S.; GONZALEZ, P.; RAMOS, A. J.; SANCHIS, V. Initial screening for inhibitory activity of essential oils on growth of Fusarium verticillioides, F. proliferatum and $F$. graminearum on maize-based agar media. Food Microbiology, Lleida, v. 21, p. 649-656, Aug. 2004. 
VELLUTI, A.; SANCHIS, V.; RAMOS, A. J.; EGIDO, J.; growth and fumonisin B production by Furarium MARÍN, S. Inhibitory effect of cinnamon, clove, proliferatum in maize grain. Food Microbiology, Lleida, v. lemongrass, orégano and palmarose essential oils on 89, p. 145-154, Jan. 2003.

Ciênc. agrotec., Lavras, v. 30, n. 4, p. 731-738, jul./ago., 2006 